\title{
ON FOURIER MULTIPLIER TRANSFORMATIONS OF BANACH-VALUED FUNCTIONS
}

\author{
BY
}

TERRY R. MCCONNELL ${ }^{1}$

\begin{abstract}
We obtain analogues of the Mihlin multiplier theorem and Littlewood-Paley inequalities for functions with values in a suitable Banach space $B$. The requirement on $B$ is that it have the unconditionality property for martingale difference sequences.
\end{abstract}

1. Introduction. A number of authors have considered the extension of the Calderón-Zygmund theory of singular integrals to vector-valued functions (see, e.g., $[\mathbf{1}, \mathbf{2}$, and 6]). Recently Burkholder [5] has obtained definitive results on transforms of Banach space-valued martingales, which are the analogues of singular integrals in probability theory, and these results have been applied to the study of singular integrals by Burkholder and McConnell [6] and by Bourgain [2 and 3]. In the present paper we use martingale methods to obtain extensions of the MihlinHörmander multiplier theorem and the Littlewood-Paley inequalities to the case of Banach-valued functions.

For suitable real-valued test functions $f$ defined on $\mathbf{R}^{n}$ let $\hat{f}$ denote the Fourier transform of $f$,

$$
\hat{f}(\lambda)=(2 \pi)^{-n} \int_{\mathbf{R}^{n}} e^{-i \lambda \cdot x} f(x) d x,
$$

and $f$ the inverse Fourier transform. To each bounded Borel function $m$ on $\mathbf{R}^{n}$ we may associate an operator $T_{m}$ defined on the test functions $f$ by

$$
T_{m} f(x)=(m \hat{f})^{\vee}(x) .
$$

The Calderón-Zygmund singular integral transformations provide an interesting class of examples (see, e.g., [22, p. 39]) and the fact that such operators are bounded on the $L^{p}$ spaces for $1<p<\infty$ suggests the following question: under what conditions on $m$ does $T_{m}$ defined in (1.2) extend to a bounded linear transformation of the $L^{p}$ spaces? A general though by no means complete answer is given by Theorem A, an improvement due to Hörmander [11] of the Mihlin multiplier theorem [17].

THEOREM A. Let $k$ be an integer greater than $n / 2$ and $m a C^{k}$ function defined on $\mathbf{R}^{n}$. Assume that there is a constant $c$ such that for all multi-indices $\alpha=$ $\left(\alpha_{1}, \ldots, \alpha_{n}\right)$ satisfying $|\alpha|=\left|\alpha_{1}\right|+\cdots+\left|\alpha_{n}\right| \leq k$ we have

$$
|\lambda|^{|\alpha|}\left|\partial^{\alpha} m(\lambda) / \partial \lambda^{\alpha}\right| \leq c .
$$

Received by the editors August 29, 1983. The results of this paper were announced at the 808th meeting of the AMS, Evanston, November 11-12, 1983.

1980 Mathematics Subject Classification. Primary 42A45, 46E40; Secondary 42A61.

Key words and phrases. Fourier multiplier, martingale transform, $L^{p}$ inequalities, vector-valued function, unconditionality.

${ }^{1}$ Supported in part by NSF Grant MCS 82-02286. 
Then for $1<p<\infty$ the operator $T_{m}$ admits an extension to $L^{p}\left(\mathbf{R}^{n}\right)$ satisfying

$$
\left\|T_{m} f\right\|_{p} \leq c_{p}\|f\|_{p}
$$

where the $c_{p}$ are constants depending only on $n, p$ and $c$.

There seem to be two distinct standard methods of proving Theorem A and related results. In the first approach, inequality (1.4) is obtained for the wider class of convolution operators whose kernel $K$ satisfies Hörmander's condition

$$
\int_{|x-y|>2|y|}|K(x-y)-K(x)| d x \leq c .
$$

Condition (1.5) implies the boundedness of the Fourier transform of $K$, and (1.4) for $p=2$ then follows by Plancherel's identity. The extension to other values of $p$ is accomplished with the aid of the Calderón-Zygmund decomposition. The second approach relies on inequalities for the Littlewood-Paley $g$-functions and, again, Plancherel's identity in the case $p=2$ plays a pivotal role. For an exposition of both of these methods of proof see [8].

Let $B$ be a real or complex Banach space and let $L^{p}\left(\mathbf{R}^{n} ; B\right)$ denote the space of $B$-valued Bochner integrable functions $f$ defined on $\mathbf{R}^{n}$ and satisfying

$$
\|f\|_{p}^{p}=\int_{\mathbf{R}^{n}}|f(x)|_{B}^{p} d x<\infty,
$$

where $|\cdot|_{B}$ denotes the norm of $B$. For functions $f$ in $L^{1}\left(\mathbf{R}^{n} ; B\right) \cap L^{\infty}\left(\mathbf{R}^{n} ; B\right)$ with finite-dimensional range we may define the Fourier transform as in (1.1) and the usual Fourier inversion formula holds. Given a bounded real-valued function $m$, define $T_{m} f$ as in (1.2). One of the goals of this paper is to show that for $B$-valued functions $f$ the conclusion (1.4) of Theorem A holds under a strengthened version of (1.3) for a large class of Banach spaces $B$. The class of Banach spaces, to be described fully below, includes all the $L^{p}$ spaces for $1<p<\infty$. However, the classical methods of proof described above break down unless $B$ has an equivalent norm under which it is a Hilbert space. The difficulty is that the Fourier transform is not, in general, a bounded operator on $L^{2}\left(\mathbf{R}^{n} ; B\right)$.

The allowable Banach spaces in our version of Theorem $A$ are the $\zeta$-convex spaces introduced by Burkholder [5]. A real or complex Banach space $B$ is $\zeta$-convex if there exists on $B \times B$ a real-valued function $\varsigma$ having the following properties:

$$
\begin{gathered}
\varsigma(x, \cdot) \text { is convex for each } x \in B, \\
\varsigma(x, y)=\varsigma(y, x), \\
\varsigma(x, y) \leq|x+y|_{B} \quad \text { if }|x|_{B} \leq 1 \leq|y|_{B},
\end{gathered}
$$

and

$$
\varsigma(0,0)>0 .
$$

For example, the spaces $L^{p}(\mathbf{R} ; \mathbf{R})$ and $l^{p}$ are $\zeta$-convex for $1<p<\infty$, while $L^{1}(\mathbf{R} ; \mathbf{R})$ and $l^{1}$ are not (see [5] for these facts and much further information concerning these spaces).

In the light of recent results, including those of the present paper, it appears likely that $\zeta$-convexity is the correct condition to impose on a Banach space $B$ in order 
for the results of classical harmonic analysis to carry over to the $B$-valued case. For example, the results of Burkholder and McConnell [6] and of Bourgain [3] taken together show that the Hilbert transform is a bounded operator on $L^{p}(\mathbf{R} ; B)$ if and only if $B$ is $\zeta$-convex. (We obtain here an independent proof that $\zeta$-convexity implies boundedness of the Hilbert transform on $L^{p}(\mathbf{R} ; B)$. See Remark 3.1 below.) More recently, Bourgain has obtained results which imply the full analogue of Theorem A for $\zeta$-convex Banach lattices [2].

Let us turn now to the precise statements of our results.

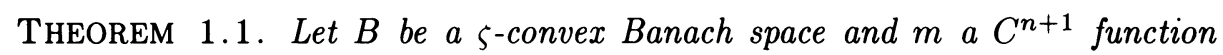
defined on $\mathbf{R}^{n}$. Assume there is a constant $c_{m}$ such that for all multi-indices $\alpha$ satisfying $|\alpha| \leq n+1$ we have

$$
|\lambda|^{|\alpha|}\left|\partial^{\alpha} m(\lambda) / \partial \lambda^{\alpha}\right| \leq c_{m}
$$

Then for $1<p<\infty$ the operator $T_{m}$ has an extension to $L^{p}\left(\mathbf{R}^{n} ; B\right)$ which satisfies

$$
\left\|T_{m} f\right\|_{p} \leq c_{p}\|f\|_{p}, \quad 1<p<\infty .
$$

The constants $c_{p}$ depend only on $c_{m}, n$ and $p$.

Note that (1.10) is more restrictive than (1.3) in Theorem A. We have been able to weaken (1.10) only at the expense of imposing the additional assumption that $m$ have support in some dyadic rectangle (a dyadic rectangle is a rectangle of the form $\left[k_{1} 2^{-l_{1}},\left(k_{1}+1\right) 2^{-l_{1}}\right] \times \cdots \times\left[k_{n} 2^{-l_{n}},\left(k_{n}+1\right) 2^{-l_{n}}\right]$ for integers $k_{1}, \ldots, k_{n}$ and $\left.l_{1}, \ldots, l_{n}\right)$.

THEOREM 1.2. Let $B$ be a $\zeta$-convex Banach space and $k$ an integer greater than $n / 2$. Let $m$ be a $C^{k}$ function on $\mathbf{R}^{n} \backslash\{0\}$ such that (1.10) holds for all multiindices $\alpha$ with $|\alpha| \leq k$. Assume, in addition, that $m$ is supported in some dyadic rectangle. Then, for $1<p<\infty$ the operator $T_{m}$ has an extension to $L^{p}\left(\mathbf{R}^{n} ; B\right)$ which satisfies (1.11).

We suspect that the conclusion of Theorem 1.2 remains true even without the assumption on the support of $m$, but we have been unable to prove this. (However, see Remark 3.2 below where we obtain a new proof of Theorem A.) A more general open question is whether the convolution operators with kernel satisfying Hörmander's condition (1.5) are bounded operators on $L^{p}\left(\mathbf{R}^{n} ; B\right)$ for $1<p<\infty$ and $B$ s-convex.

Our final result is an extension of the Littlewood-Paley inequalities to the $B$ valued case. To decribe the classical results fix an ordering of the dyadic rectangles $\left\{I_{k}\right\}_{k \geq 1}$, and, for $f$ in $L^{1}\left(\mathbf{R}^{n} ; B\right) \cap L^{\infty}\left(\mathbf{R}^{n} ; B\right)$ having finite-dimensional range, define the Littlewood-Paley function $S(f)$ by

$$
S(f)(x)=\left(\sum_{k=1}^{\infty}\left|f_{k}(x)\right|_{B}^{2}\right)^{1 / 2}
$$

where $f_{k}(x)=\int_{I_{k}} \hat{f}(\lambda) e^{i \lambda \cdot x} d \lambda$. In the real case $(B=\mathbf{R})$ Littlewood and Paley $[\mathbf{1 4}$, 15 and 16] proved that there are constants $c_{p}$ depending only on $n$ and $p$ such that

$$
c_{p}^{-1}\|f\|_{p} \leq\|S(f)\|_{p} \leq c_{p}\|f\|_{p}
$$


for $1<p<\infty$. Let $\left(\varepsilon_{k}\right)_{k \geq 1}$ be any sequence of numbers from $\{ \pm 1\}$ and define an operator $T_{(\varepsilon)}$ by

$$
T_{(\varepsilon)} f(x)=\sum_{k=1}^{\infty} \varepsilon_{k} f_{k}(x) .
$$

An immediate consequence of (1.12) in the real case is that $T_{(\varepsilon)}$ satisfies

$$
c_{p}^{-1}\|f\|_{p} \leq\left\|T_{(\varepsilon)} f\right\|_{p} \leq c_{p}\|f\|_{p}
$$

for $1<p<\infty$. In fact, Khintchine's inequalities imply that (1.12) and (1.13) are equivalent.

The situation in the $B$-valued case is quite different: Inequalities (1.12) hold if and only if $B$ is isomorphic to a subspace of a Hilbert space, while inequalities (1.13) hold if and only if $B$ is $\zeta$-convex. The latter statement is Theorem 1.3 and the first may be seen as follows: first, it is not difficult to show that (1.12) implies that for all finite sequences of vectors $x_{1}, \ldots, x_{N}$ chosen from $B$ we have

$$
\frac{1}{c} \sum_{k=1}^{N}\left|x_{k}\right|_{B}^{2} \leq \int_{-\pi}^{\pi}\left|\sum_{k=1}^{N} x_{k} e^{2^{k} i x}\right|_{B}^{2} d x \leq c \sum_{k=1}^{N}\left|x_{k}\right|_{B}^{2} .
$$

It then follows from the central limit theorem for lacunary Fourier series [20] that if $\xi_{1}, \xi_{2}, \ldots, \xi_{N}$ are independent identically distributed standard Gaussian random variables, then there is a constant $c$ such that, for any sequence $x_{k}$ as above,

$$
\frac{1}{c} \sum_{k=1}^{N}\left|x_{k}\right|_{B}^{2} \leq E\left|\sum_{k=1}^{N} x_{k} \xi_{k}\right|_{B}^{2} \leq c \sum_{k=1}^{N}\left|x_{k}\right|_{B}^{2} .
$$

Here $E$ denotes mathematical expectation with respect to the $\xi_{k}$. Finally, Kwapień [13] has shown that inequalities (1.14) hold only if $B$ is isomorphic to a Hilbert space. (For the fact that inequalities (1.12) do hold in case $B$ is a Hilbert space see [8].)

THEOREM 1.3. Let $T_{(\varepsilon)}$ be defined as above for any sequence $\varepsilon=\left(\varepsilon_{k}\right)_{k \geq 1}$ of numbers chosen from $\{ \pm 1\}$. The inequalities (1.13) hold for $B$-valued functions $f$ if and only if $B$ is 5 -convex.

The proofs of these theorems use a number of ideas and results from probability and therefore we have devoted the second section of this paper to an exposition of some background material. The third and final section contains the proofs of Theorems 1.1-1.3.

Throughout the paper we will denote by $D_{F}\left(\mathbf{R}^{n} ; B\right)$ and $S_{F}\left(\mathbf{R}^{n} ; B\right)$ the spaces of functions defined on $\mathbf{R}^{n}$, taking values in some fixed finite-dimensional subspace of $B$, and with each component function belonging, respectively, to the space of $C^{\infty}$ functions of compact support and the space of Schwartz test functions. We shall sometimes omit $F$ and $B$ when $B$ equals $\mathbf{R}$. The notation does not specify in which finite-dimensional subspace of $B$ the functions belonging to these spaces take their values, but this should cause no confusion. We shall repeatedly use the fact that the union of these spaces over all finite-dimensional subspaces of $B$ forms a dense subset of $L^{p}\left(\mathbf{R}^{n} ; B\right)$ for each $0<p<\infty$.

The letter $c$ will stand for a constant, possibly different from one usage to the next. Often $c$ will carry a subscript to emphasize its dependence on a particular 
parameter. If the subscript is the number of a statement then the constant is the same as the constant appearing in that numbered statement.

2. Some preliminary results from probability theory. The purpose of this section is to present some background material from probability theory. Much of this is known, though perhaps not in the form presented here. For basic notions concerning Brownian motion and martingale theory see [12 and 18].

Let $(\Omega, \mathcal{F}, P)$ be a probability space, $B$ a Banach space, and $\left(\mathcal{F}_{k}\right)_{k \geq 0}$ an increasing sequence of sub- $\sigma$-fields of $\mathcal{F}$. A sequence $\left(d_{k}\right)$ of Bochner integrable, $\mathcal{F}_{k}$-measurable random variables is a martingale difference sequence relative to $\mathcal{F}_{k}$ provided that $E\left(d_{k} \mid F_{k-1}\right)=0$, almost surely, for $k \geq 1$. The Banach space $B$ has the unconditionality property for martingale differences (UMD) if, for some $1<p<\infty$, each such martingale difference sequence forms an unconditional basis for the subspace of $L^{p}(\Omega ; B)$ which it spans. Burkholder [5] has shown that the class of UMD Banach spaces coincides with the class of $\zeta$-convex spaces and also with the class of Banach spaces $B$ for which the transform inequalities of $[4]$ for real-valued martingales carry over to $B$-valued martingales. This latter result is a key tool in the present work and we therefore describe it in more detail. Given a sequence $\left(e_{k}\right)_{k \geq 0}$ of bounded real-valued random variables, with $e_{k}$ being $\mathcal{F}_{k-1}$-measurable for $k \geq 1$ and with $e_{0}=1$, and given a $B$-valued martingale difference sequence $\left(d_{k}\right)$, the sequence $\left(e_{k} d_{k}\right)$ is another martingale difference sequence called the transform of $d_{k}$ by $e_{k}$. Then [5], $B$ is UMD if and only if there are constants $c_{p}(B)$, depending only on $B$ and $1<p<\infty$, such that for each $N$ and all such sequences $\left(e_{k}\right)$ and $\left(d_{k}\right)$ with the $e_{k}$ bounded by one we have

$$
\left\|\sum_{i=0}^{N} e_{i} d_{i}\right\|_{p} \leq c_{p}(B)\left\|\sum_{i=0}^{N} d_{i}\right\|_{p}
$$

Many martingales of interest in harmonic analysis arise in connection with Brownian motion. We shall be concerned here with $(n+1)$-dimensional Brownian motion $Z_{t}$ in the half-space $\mathbf{R}_{+}^{n+1}=\left\{(x, y): x \in \mathbf{R}^{n}\right.$ and $\left.y>0\right\}$, and we will use the component notation $Z_{t}=\left(X_{t}, Y_{t}\right)$, where $Y_{t}$ is one-dimensional Brownian motion and $X_{t}$ is an independent $n$-dimensional Brownian motion. As usual in the theory of Markov processes the expression $P_{(x, y)}$ refers to the probability measure governing $Z_{t}$ when the initial value is $(x, y)$, and $E_{(x, y)}$ to the corresponding expectation.

Let $X(y), y>0$, denote the value of $X_{t}$ at the first time $Y_{t}$ takes a value less than or equal to $y$. The random variable $X(y)$ has probability density function given in terms of the Poisson kernel by

$$
\frac{P_{\left(x^{\prime}, y^{\prime}\right)}(X(y) \in d x)}{d x}=c_{n} \frac{\left(y^{\prime}-y\right)}{\left(\left|x-x^{\prime}\right|^{2}+\left(y^{\prime}-y\right)^{2}\right)^{(n+1) / 2}},
$$

where $y^{\prime}>y$ and $c_{n}$ is the constant $\pi^{-(n+1) / 2} \Gamma((n+1) / 2)$. In the rest of the paper the right side of $(2.2)$ will be denoted by $h_{x}\left(x^{\prime}, y^{\prime}-y\right)$ and called the Cauchy density centered at $x$ with parameter $y^{\prime}-y$.

The process $X(y)$ has independent increments when considered as a stochastic process with time parameter $y$. This fact together with the Poisson integral representation of harmonic functions yields the following result. 
LEMMA 2.1. Let $u$ be a bounded harmonic function on $\mathbf{R}_{+}^{n+1}$ with values in a finite-dimensional Banach space (i.e., each component of $u$ is harmonic in the usual sense). Let $y_{N}<y_{N-1}<\cdots<y_{0}$ be given. Then for any $x \in \mathbf{R}^{n}$ the sequence $\left(d_{i}\right)$ with $d_{i}=u\left(X\left(y_{i}\right), y_{i}\right)-u\left(X\left(y_{i-1}\right), y_{i-1}\right), i \geq 1$, and $d_{0}=u\left(x, y_{0}\right)$ is a martingale difference sequence under the measure $P_{\left(x, y_{0}\right)}$ relative to an appropriate sequence of $\sigma$-fields $\mathcal{F}_{k}$. The $\sigma$-field $\mathcal{F}_{k}$ may be chosen as the smallest $\sigma$-field with respect to which $d_{0}, d_{1}, \ldots, d_{k}$ are measurable.

Doob [7] has shown that there is a family of Markov processes corresponding to the intuitive notion of Brownian motion conditioned to exit $\mathbf{R}_{+}^{n+1}$ at given points. More precisely, for $\xi \in \mathbf{R}^{n}$ there are strong Markov processes $Z_{t}^{/ \xi}=\left(X_{t}^{/ \xi}, Y_{t}^{/ \xi}\right)$, defined on a common probability space $(\Omega, \mathcal{F}, P)$ with $Z_{t}$, having continuous paths up to time $\zeta=\inf \left\{t: Y_{t}^{/ \xi}=0\right\}$, and having the following additional properties $\left(P_{(x, y)}^{/ \xi}\right.$ denotes as usual the measure corresponding to initial value $\left.(x, y)\right)$ :

(i) $P_{(x, y)}^{/ \xi}\left(X_{\zeta-}^{/ \xi}=\xi\right)=1$.

(ii) The family $P_{(x, y)}^{/ \xi}$ gives a regular version of the conditional probabilities $P_{(x, y)}\left(\cdot \mid X_{\zeta-}=\xi\right)$ as $\xi$ ranges over $\mathbf{R}^{n}$, i.e., for any Borel subset $D$ of $\mathbf{R}^{n}$, and bounded $\mathcal{F}$-measurable random variable $W$ we have

$$
E_{(x, y)}\left(W ; X_{\zeta^{-}} \in D\right)=\int_{D} E_{(x, y)}^{/ \xi}(W) h_{\xi}(x, y) d \xi
$$

(iii) For any bounded Borel function $F$ and $0<y_{1}<y$ we have

$$
E_{(x, y)}^{/ \xi} F\left(X^{/ \xi}\left(y_{1}\right)\right)=\frac{1}{h_{\xi}(x, y)} E_{(x, y)} F\left(X\left(y_{1}\right)\right) h_{\xi}\left(X\left(y_{1}\right), y_{1}\right) .
$$

In the future we shall drop the superscripts on $Z_{t}^{/ \xi}, X_{t}^{/ \xi}$ and $Y_{t}^{/ \xi}$, referring to the conditioning only through the measures $P_{(x, y)}^{/ \xi}$.

Our next result is similar to one that has been used by Gundy, Varopolous and Silverstein in their study of the Riesz transforms [9 and 10]. A more abstract version of the result is due to Rota [19].

LEMMA 2.2. Let $u$ be bounded and harmonic on $\mathbf{R}_{+}^{n+1}$ with values in a finitedimensional Banach space. Let $0<y_{1}<y_{2}<A$ and $x \in \mathbf{R}^{n}$ be given. Then

$$
u\left(x, y_{1}+y_{2}\right)=\int_{\mathbf{R}^{n}} h_{x}\left(x^{\prime}, A\right) E_{\left(x^{\prime}, A\right)}^{/ x} u\left(X\left(y_{1}\right), y_{2}\right) d x^{\prime}
$$

ProOF. By (2.4),

$$
h_{x}\left(x^{\prime}, A\right) E_{\left(x^{\prime}, A\right)}^{/ x} u\left(X\left(y_{1}\right), y_{2}\right)=E_{\left(x^{\prime}, A\right)} u\left(X\left(y_{1}\right), y_{2}\right) h_{x}\left(X\left(y_{1}\right), y_{1}\right) .
$$

The right side of (2.5) then becomes

$$
\begin{aligned}
\int_{\mathbf{R}^{n}} & E_{\left(x^{\prime}, A\right)} u\left(X\left(y_{1}\right), y_{2}\right) h_{x}\left(X\left(y_{1}\right), y_{1}\right) d x^{\prime} \\
& =\int_{\mathbf{R}^{n}} \int_{\mathbf{R}^{n}} u\left(\xi, y_{2}\right) h_{x}\left(\xi, y_{1}\right) h_{x^{\prime}}\left(\xi, A-y_{1}\right) d x^{\prime} d \xi \\
& =\int_{\mathbf{R}^{n}} u\left(\xi, y_{2}\right) h_{x}\left(\xi, y_{1}\right) d \xi=u\left(x, y_{1}+y_{2}\right),
\end{aligned}
$$

and the proof is complete. 
Denote by $P^{x, A}$ the probability measure on the sample space of $Z_{t}$ defined by

$$
P^{x, A}(D)=\int_{\mathbf{R}^{n}} h_{x}\left(x^{\prime}, A\right) P_{\left(x^{\prime}, A\right)}^{/ x}(D) d x^{\prime}
$$

for $D \in \mathcal{F}$. By the argument in the proof of the previous lemma these measures induce consistent probability distributions on $X(y)$ as $A>y$ varies. The Kolmogorov extension theorem then implies that inverse limit measures $P^{x}$ exist as $A$ tends to infinity. In terms of these measures the result of Lemma 2.2 may be expressed more concisely as

$$
u\left(x, y_{1}+y_{2}\right)=E^{x} u\left(X\left(y_{1}\right), y_{2}\right)
$$

for all positive $y_{1}$ and $y_{2}$, and for $u$ harmonic as above.

An easy consequence of (2.7) is that the variables $X(y)$ have under $P^{x}$ a Cauchy distribution centered at $x$ with parameter $y$. The final result of this section completes the description of $X(y)$ as a process indexed by $y$.

LEMMA 2.3. Under $P^{x}$ the process $X(y)$ has independent increments.

ProOF. Without loss of generality we may take $x=0$. Let $\hat{Z}_{t}$ denote a process defined on some probability space $(\hat{\Omega}, \hat{\mathcal{F}}, \hat{P})$ for which $\hat{X}_{t}$ and $\hat{Y}_{t}$ are independent, $\hat{X}_{t}$ is an $n$-dimensional Brownian motion started from 0 , and $\hat{Y}_{t}$ is a Bessel (3) diffusion started from its entrance boundary point 0 (see $[12,2.7]$ for a discussion of the Bessel (3) diffusion. Such a process may be realized as the magnitude of a 3-dimensional Brownian motion). Let $Z_{t}$ be a Brownian motion conditioned to exit from $\mathbf{R}_{+}^{n+1}$ at 0 (i.e., $Z_{t}^{/ 0}$ ) and $\varsigma$ denote the exit time. For $A>0$ let $\hat{L}_{A}$ denote the last exit time of $\hat{Y}_{t}$ from the level $y=A, \hat{L}_{A}=\sup \left\{t: \hat{Y}_{t} \leq A\right\}$. We will show that for any $A>0$ the process $Z_{\zeta-t}, 0 \leq t<\varsigma$, has the same finite-dimensional distributions under $P^{0, A}$ as has the process $\hat{Z}_{t}, 0 \leq t<\hat{L}_{A}$, under $\hat{P}$.

The equivalence of $\hat{Y}_{t}, 0 \leq t<\hat{L}_{A}$, and $Y_{\zeta-t}, 0 \leq t<\varsigma$, follows easily from a result of M. J. Sharpe [21]. In particular, $\hat{L}_{A}$ and $\zeta$ have the same distributions under $\hat{P}$ and $P^{0, A}$, respectively. This fact and an elementary calculation of Gaussian conditional probabilities gives the following string of equalities: For $D$ a Borel subset of $\mathbf{R}^{n}$,

$$
\begin{aligned}
p^{0, A} & \left(X_{\zeta-t} \in D, t<\varsigma\right)=\int_{\mathbf{R}^{n}} h_{0}\left(x^{\prime}, A\right) P_{\left(x^{\prime}, A\right)}^{/ 0}\left(X_{\zeta-t} \in D, t<\varsigma\right) d x^{\prime} \\
& =\int_{\mathbf{R}^{n}} h_{0}\left(x^{\prime}, A\right) \int_{t}^{\infty} P_{\left(x^{\prime}, A\right)}^{0}(\varsigma \in d s) P_{\left(x^{\prime}, A\right)}\left(X_{s-t} \in D \mid X_{s}=0\right) d x^{\prime} \\
& =\int_{\mathbf{R}^{n}} h_{0}\left(x^{\prime}, A\right) \int_{t}^{\infty} \hat{P}\left(\hat{L}_{A} \in d s\right) \hat{P}\left(\hat{X}_{t} \in D \mid \hat{X}_{s}=x^{\prime}\right) d x^{\prime} .
\end{aligned}
$$

Since the process $\hat{X}_{s}$ is independent of $\hat{L}_{A}$ and $h_{0}\left(x^{\prime}, A\right)$ is exactly the probability density function of $\hat{X}_{\hat{L}_{A}}$, the last expression reduces to $\hat{P}\left(\hat{X}_{t} \in D, t<\hat{L}_{A}\right)$. Similarly one obtains the equality of the higher order finite-dimensional distributions.

Since $A$ is arbitrary the proof now reduces to checking the independence of the increments of $\hat{X}_{\hat{L}_{y}}$, considered as a process indexed by $y$, for $0 \leq y<A$. But this is immediate from the fact that the families $\left(\hat{X}_{s}\right)$ and $\left(\hat{L}_{y}\right)$, the later being defined in terms of $\hat{Y}_{t}$ only, are independent. 
3. Proofs of the theorems. For the proof of Theorem 1.1 it is convenient first to reduce to the case of sufficiently regular functions $f$ and multipliers $m$. Therefore let $m$ be a function belonging to $D\left(\mathbf{R}^{n}\right)$, vanishing in a neighborhood of the origin, and satisfying

$$
|\lambda|^{\alpha}\left|\partial^{\alpha} m(\lambda) / \partial \lambda^{\alpha}\right| \leq c_{m}, \quad|\alpha| \leq n+1,
$$

for some constant $c_{m}$. We shall prove below that there are constants $c_{p}$, depending only on $n, c_{m}$ and $p$, such that

$$
\left\|T_{m} f\right\|_{p} \leq c_{p}\|f\|_{p}
$$

for all $f \in D_{F}\left(\mathbf{R}^{n} ; B\right)$. Here let us show how Theorem 1.1 follows.

Suppose first that $m$ satisfies (3.1), has compact support, and vanishes in a neighborhood of the origin, but is not assumed to be $C^{\infty}$. It is then possible to find a sequence of functions $\left(m_{k}\right) \subseteq D\left(\mathbf{R}^{n}\right)$ converging uniformly to $m$, each vanishing near the origin and satisfying (3.1) with $c_{m}$ replaced by $2 c_{m}$. The $m_{k}$ may be realized as convolutions of $m$ with an appropriate sequence of approximate identities. The Fourier inversion formula shows that $T_{m_{k}} f(x)$ converges to $T_{m} f(x)$ for each $x$ in $\mathbf{R}^{n}$. Since, by assumption, (3.2) applies to each $m_{k}$, the theorem follows for such special $m$.

For the general case choose smooth functions $\psi$ and $\phi$ with $\psi$ supported in $|\lambda| \leq 1$ and satisfying $\psi(\lambda)=1$ for $|\lambda| \leq \frac{1}{2}$, and with $\phi$ such that $\phi(\lambda)=1$ for $|\lambda| \geq 1$ and $\phi(\lambda)=0$ for $|\lambda| \leq \frac{1}{2}$. One shows with the aid of Leibniz's formula that the multipliers $\mu_{k}(\lambda)=\psi(\lambda / k) \phi(k \lambda) m(\lambda)$ satisfy (3.1) with a constant independent of $k$. The $\mu_{k}$ converge boundedly to $m$ on $\mathbf{R}^{n} \backslash\{0\}$ and the Fourier inversion formula again shows that $T_{\mu_{k}} f$ converges pointwise to $T_{m} f$ for $f \in D_{F}\left(\mathbf{R}^{n} ; B\right)$. Since the inequality (3.2) holds for the $\mu_{k}$ it holds as well for $m$. Finally, since the $D_{F}\left(\mathbf{R}^{n} ; B\right)$ are dense in $L^{p}\left(\mathbf{R}^{n} ; B\right)$ for each $1<p<\infty$, it follows that $T_{m}$ has a bounded extension to $L^{p}\left(\mathbf{R}^{n} ; B\right)$ which also satisfies (3.2).

For the remainder of this section, except where noted otherwise, we shall assume that $m$ satisfies (3.1) and the hypotheses preceding (3.1).

Let $M$ denote the inverse Fourier transform of $m$. Then $M \in S\left(\mathbf{R}^{n}\right)$ and we have that $T_{m} f(x)=M * f(x)$ for each $f \in D_{F}\left(\mathbf{R}^{n} ; B\right)$. Since $M * f$ then belongs to $S_{F}\left(\mathbf{R}^{n} ; B\right)$, the Poisson integrals of $f, M$ and $T_{m} f$, denoted respectively by $u$, $v$ and $w$, exist and are harmonic functions on $\mathbf{R}_{+}^{n+1}$.

Our starting point for the proof of (3.2) is the following representation of $T_{m} f$ :

$$
T_{m} f(x)=\frac{(-4)^{n+2}}{(n+1) !} \int_{0}^{\infty} \int_{\mathbf{R}^{n}} y^{n+1} v^{(n+1)}(s, y) u_{y}(x-s, 3 y) d s d y
$$

(Here and hereafter $v^{(n+1)}$ denotes the $(n+1)$-fold partial derivative of $v$ with respect to $y$.) To prove (3.3) we proceed as in [22]. The semigroup property of the Poisson integral implies

$$
w\left(x, y_{1}+y_{2}\right)=\int_{\mathbf{R}^{n}} v\left(s, y_{1}\right) u\left(x-s, y_{2}\right) d s
$$

for each pair of nonnegative numbers $y_{1}$ and $y_{2}$. After $n+1$ integrations by parts and a change of variables in the formula

$$
T_{m} f(x)=-\int_{0}^{\infty} w_{y}(x, y) d y
$$


we obtain

$$
\begin{aligned}
T_{m} f(x) & =\frac{(-1)^{n+2}}{(n+1) !} \int_{0}^{\infty} y^{n+1} w^{(n+2)}(x, y) d y \\
& =\frac{(-4)^{n+2}}{(n+1) !} \int_{0}^{\infty} y^{n+1} w^{(n+2)}(x, 4 y) d y .
\end{aligned}
$$

Differentiating (3.4) $n+1$ times with respect to $y_{1}$, once with respect to $y_{2}$, and then setting $y_{1}$ equal to $y$ and $y_{2}$ equal to $3 y$ yields (3.3).

For any $A>0$ define $T_{m}^{A} f(x)$ by the following truncated version of (3.3):

$$
T_{m}^{A} f(x)=\frac{(-4)^{n+2}}{(n+1) !} \int_{0}^{A} \int_{\mathbf{R}^{n}} y^{n+1} v^{(n+1)}(s, y) u_{y}(x-s, 3 y) d s d y .
$$

It suffices to prove (3.2) with $T_{m} f$ replaced by $T_{m}^{A} f$ and $c_{p}$ independent of $A$. Therefore fix such a positive number $A$ for the rest of the proof.

The next step is to replace the integral with respect to $y$ in the definition of $T_{m}^{A} f$ with a Riemann sum approximation. Let $\Pi_{N}$ denote a partition of $[0, A]$ defined by choosing points $y_{i}$ satisfying $0=y_{0}<y_{1}<\cdots<y_{N}=A$. We suppose that this is done in such a way that $\Pi_{N+1}$ refines $\Pi_{N}$ and that the mesh size $\mu_{N}$ is bounded by a multiple of $N^{-1}$. Define

$$
g_{N}(x)=\sum_{i=0}^{N-1} \int_{\mathbf{R}^{n}} y_{i}^{n+1} v^{(n+1)}\left(s, y_{i}\right)\left[u\left(x-s, 2 y_{i+1}+y_{i}\right)-u\left(x-s, 3 y_{i}\right)\right] d s
$$

In view of the smoothness assumptions made above it is not hard to show that for each $x$ in $\mathbf{R}^{n}$ we have

$$
\lim _{N \rightarrow \infty} g_{N}(x)=\frac{2(-4)^{n+1}}{(n+1) !} T_{m}^{A} f(x) .
$$

A sketch of the argument follows. Set $\rho(s, y)=y^{n+1} v^{(n+1)}(s, y)$. By the second mean value theorem for integrals there is for each fixed $s$ and $i \leq N-1$ a point $y^{*}$ in $\left[y_{i}, y_{i+1}\right]$ such that

$$
\begin{aligned}
\int_{y_{i}}^{y_{i+1}} & \rho(s, y) u_{y}\left(x-s, y_{i}+2 y\right) d y \\
= & \left.\frac{1}{2} \rho\left(s, y^{*}\right) u\left(x-s, y_{i}+2 y_{i+1}\right)-u\left(x-s, 3 y_{i}\right)\right]
\end{aligned}
$$

Therefore

$$
\begin{aligned}
\int_{y_{i}}^{y_{i+1}} & \rho(s, y) u_{y}(x-s, 3 y) d y \\
= & \frac{1}{2} \rho\left(s, y^{*}\right)\left[u\left(x-s, y_{i}+2 y_{i+1}\right)-u\left(x-s, 3 y_{i}\right)\right] \\
& +\int_{y_{i}}^{y_{i+1}} \rho(s, y)\left[u_{y}(x-s, 3 y)-u_{y}\left(s-x, y_{i}+2 y\right)\right] d y
\end{aligned}
$$

Using the mean value theorem the last expression may be written as

$$
\frac{1}{2} \rho\left(s, y_{i}\right)\left[u\left(x-s, 2 y_{i+1}+y_{i}\right)-u\left(x-s, 3 y_{i}\right)\right]+\varepsilon(s, x, N, i)
$$

where the error satisfies

$$
|\varepsilon(s, x, N, i)|_{B} \leq \mu_{N}^{2} \gamma(s)
$$


and $\gamma$ is an integrable function of $s$ depending on $A, x, m$ and $f$, but not on $i$. Therefore there is a constant $C$ independent of $N$ such that

$$
\int_{\mathbf{R}^{n}} \sum_{i=0}^{N-1}|\varepsilon(s, x, N, i)|_{B} d s \leq C N \mu_{N}^{2}
$$

The convergence of $g_{N}(x)$ follows since $N \mu_{N}$ is bounded, $\mu_{N}$ approaches zero, and the left side of (3.6) dominates the difference

$$
\left|g_{N}(x)-\frac{2(-4)^{-(n+2)}}{(n+1) !} T_{m}^{A} f(x)\right|_{B} .
$$

Thus it is sufficient to show that there are constants $c_{p}$ depending only on $n, c_{m}$ and $p$ such that

$$
\left\|g_{N}\right\|_{p} \leq c_{p}\|f\|_{p}
$$

The proof of (3.7) uses a probabilistic representation of $g_{N}(x)$ which is based on Lemma 2.2. Taking $y_{1}=y_{i+1}$ and $y_{2}=y_{i}+y_{i+1}$ in (2.7) we obtain

$$
u\left(x-s, y_{i}+2 y_{i+1}\right)=E^{x} u\left(X\left(y_{i+1}\right)-s, y_{i}+y_{i+1}\right) .
$$

Similarly

$$
u\left(x-s, 3 y_{i}\right)=E^{x} u\left(X\left(y_{i}\right)-s, 2 y_{i}\right) .
$$

Define $e(s, y)$ by

$$
e(s, y)=y^{n+1} v^{(n+1)}(s, y) h_{0}^{-1}(s, y),
$$

where $h_{0}(s, y)$ is the Cauchy density (Poisson kernel) centered at 0 . Let $\tilde{Z}_{t}=$ $\left(\tilde{X}_{t}, \tilde{Y}_{t}\right)$ be a Brownian motion, independent of $Z_{t}$, with associated probability measures $\tilde{P}_{(x, y)}$ and $\tilde{P}^{x}$. Since $h_{0}(s, y)$ is the density function of $\tilde{X}(y)$ under $\tilde{P}^{0}$, the division by $h_{0}$ in (3.8) allows us to express the $s$-integration in (3.5) as an expectation with respect to $\tilde{P}^{0}$. This leads to the following probabilistic representation of $g_{N}$ :

$$
\begin{aligned}
g_{N}(x)=\tilde{E}^{0} E^{x} \sum_{i=0}^{N-1} e\left(\tilde{X}\left(y_{i}\right), y_{i}\right)\left[u\left(X\left(y_{i+1}\right)-\tilde{X}\left(y_{i}\right), y_{i+1}+y_{i}\right)\right. & \\
& \left.-u\left(X\left(y_{i}\right)-\tilde{X}\left(y_{i}\right), 2 y_{i}\right)\right] .
\end{aligned}
$$

It is convenient to abbreviate the expression in brackets as $(\Delta u)_{i}$. For each fixed point in the sample space of $\tilde{Z}_{t}$ an application of the Poisson integral formula to the translate of $u$ by $\tilde{X}\left(y_{i}\right)$ (see also Lemma 2.1) shows that the $(\Delta u)_{i}$ form a martingale difference sequence under $P_{\left(x^{\prime}, A\right)}$ for each $x^{\prime} \in \mathbf{R}^{n}$.

By Jensen's inequality for $1<p<\infty$,

$$
\int_{\mathbf{R}^{n}}\left|g_{N}(x)\right|_{B}^{p} d x \leq \int_{\mathbf{R}^{n}} \tilde{E}^{0} E^{x}\left|\sum_{i=0}^{N-1} e\left(\tilde{X}\left(y_{i}\right), y_{i}\right)(\Delta u)_{i}\right|_{B}^{p} d x .
$$


Using the definition of $P^{x}$ (which may be replaced by $P^{x, A}$ in the calculation) and (2.3) in performing the $x$ integration we obtain

$$
\begin{aligned}
\int_{\mathbf{R}^{n}}\left|g_{N}(x)\right|_{B}^{p} d x & \leq \int_{\mathbf{R}^{n}} \tilde{E}^{0} \int_{\mathbf{R}^{n}} h_{x}\left(x^{\prime}, A\right) E_{\left(x^{\prime}, A\right)}^{\prime x}\left|\sum_{i=0}^{N-1} e\left(\tilde{X}\left(y_{i}\right), y_{i}\right)(\Delta u)_{i}\right|_{B}^{p} d x^{\prime} d x \\
& =\int_{\mathbf{R}^{n}} \tilde{E}^{0} E_{\left(x^{\prime}, A\right)}\left|\sum_{i=0}^{N-1} e\left(\tilde{X}\left(y_{i}\right), y_{i}\right)(\Delta u)_{i}\right|_{B}^{p} d x^{\prime} .
\end{aligned}
$$

The next step is to apply Burkholder's inequality (2.1) to the $P_{\left(x^{\prime}, A\right)}$ expectation with $e_{i}=e\left(\tilde{X}\left(y_{i}\right), y_{i}\right)$. The following lemma gives the required uniform bound on the $e_{i}$.

LEMMA 3.1. There is a constant $c$ depending only on $n$ and $c_{m}$ such that $|e(s, y)| \leq c$ for all $s \in \mathbf{R}^{n}$ and $y>0$.

PROOF. The Fourier inversion formula gives the following expression for $e(s, y)$ :

$$
e(s, y)=\left(|s|^{2}+y^{2}\right)^{(n+1) / 2} y^{n} \int_{\mathbf{R}^{n}} e^{i \lambda \cdot s} m(\lambda)|\lambda|^{n+1} e^{-|\lambda| y} d \lambda .
$$

There are two cases to consider: $|s|<y$ and $|s| \geq y$. In the first case we have

$$
\begin{aligned}
|e(s, y)| & \leq c_{n} y^{2 n+1} \int_{\mathbf{R}^{n}}|m(\lambda)||\lambda|^{n+1} e^{-|\lambda| y} d \lambda \\
& \leq c_{n} c_{m} y^{2 n+1} \int_{0}^{\infty} r^{2 n} e^{-r y} d r \\
& =c_{n} c_{m} \int_{0}^{\infty} r^{2 n} e^{-r} d r
\end{aligned}
$$

which is a constant depending only on $n$ and $m$.

For the second case let $\nabla$ denote the gradient operating on functions of $\lambda$. Then

$$
\begin{aligned}
|e(s, y)| & \leq\left. c_{n}|s|^{n+1} y^{n}\left|\int_{\mathbf{R}^{n}} e^{i \lambda \cdot s} m(\lambda)\right| \lambda\right|^{n+1} e^{-|\lambda| y} d \lambda \mid \\
& =\left.c_{n} y^{n}\left|\int_{\mathbf{R}^{n}}\left[\left(\frac{s}{|s|} \cdot \nabla\right)^{n+1} e^{i \lambda \cdot s}\right] m(\lambda)\right| \lambda\right|^{n+1} e^{-|\lambda| y} d \lambda \mid
\end{aligned}
$$

which, after integration by parts, becomes

$$
\left.c_{n} y^{n}\left|\int_{\mathbf{R}^{n}} e^{i \lambda \cdot s}\left(\frac{s}{|s|} \cdot \nabla\right)^{n+1} m(\lambda)\right| \lambda\right|^{n+1} e^{-|\lambda| y} d \lambda \mid .
$$

Expansion of the operator $(s /|s| \cdot \nabla)^{n+1}$ produces many terms, each of which may be estimated in a similar fashion. For example, one of the terms is

$$
\left.c_{n} y^{n}\left|\int_{\mathbf{R}^{n}} e^{i \lambda \cdot s}\left(\frac{\partial}{\partial \lambda_{1}}\right)^{n+1} m(\lambda)\right| \lambda\right|^{n+1} e^{-|\lambda| y} d \lambda \mid
$$

which may be estimated via Leibniz's formula in terms of expressions of the form

$$
\left.y^{n} \int_{\mathbf{R}^{n}}\left|\left(\frac{\partial}{\partial \lambda_{1}}\right)^{\alpha} m(\lambda)\left(\frac{\partial}{\partial \lambda_{1}}\right)^{\beta}\right| \lambda\right|^{n+1}\left(\frac{\partial}{\partial \lambda_{1}}\right)^{\gamma} e^{-|\lambda| y} \mid d \lambda
$$


with $\alpha+\beta+\gamma=n+1$. (Recall that $m$ vanishes in a neighborhood of the origin so that there is no difficulty differentiating $|\lambda|$.) Since $|\lambda|^{\alpha}\left|\left(\partial / \partial \lambda_{1}\right)^{\alpha} m(\lambda)\right| \leq c_{m}$, the last expression is bounded by

$$
c_{n} c_{m} y^{n} \int_{\mathbf{R}^{n}}|\lambda|^{\gamma}\left|\left(\frac{\partial}{\partial \lambda_{1}}\right)^{\gamma} e^{-|\lambda| y \mid}\right| d \lambda \leq c_{n} c_{m} y^{n+\gamma} \int_{0}^{\infty} r^{n+\gamma-1} e^{-r y} d r
$$

which, as above, is a constant having the desired dependence.

Returning to the proof of Theorem 1.1, apply (2.1) in (3.10) to obtain

$$
\begin{aligned}
\int_{\mathbf{R}^{n}}\left|g_{N}(x)\right|_{B}^{p} & \leq c_{p} \int_{\mathbf{R}^{n}} \tilde{E}^{0} E_{\left(x^{\prime}, A\right)}\left|\sum_{i=0}^{N-1}(\Delta u)_{i}\right|_{B}^{p} d x^{\prime} \\
& =c_{p} \int_{\mathbf{R}^{n}} \tilde{E}^{0} E^{x}\left|\sum_{i=0}^{N-1}(\Delta u)_{i}\right|_{B}^{p} d x .
\end{aligned}
$$

This last written quantity contains no dependence on the multiplier $m$, but has yet to be related to $f$, the boundary-value function of $u$. For that we use the following

LEMMA 3.2. The sequence $X\left(y_{0}\right)-\tilde{X}\left(y_{0}\right), X\left(y_{1}\right)-\tilde{X}\left(y_{0}\right), X\left(y_{1}\right)-\tilde{X}\left(y_{1}\right), \ldots$, $X\left(y_{N}\right)-\tilde{X}\left(y_{N}\right)$ has the same distribution under the product measure $\tilde{P}^{0} \otimes P^{x}$ as has the sequence $X\left(2 y_{0}\right), X\left(y_{0}+y_{1}\right), X\left(2 y_{1}\right), \ldots, X\left(2 y_{N}\right)$ under $P^{x}$.

Proof. Put $\xi_{j}=X\left(y_{j}\right)-X\left(y_{j-1}\right)$ and $\tilde{\xi}_{j}=\tilde{X}\left(y_{j}\right)-\tilde{X}\left(y_{j-1}\right)$ for $j=$ $1,2, \ldots, N$. Also put $\xi_{0}=x$ and $\tilde{\xi}_{0}=0$. Under $\tilde{P}^{0} \otimes P^{x}$ the sequences $\left(\xi_{j}\right)$ and $\left(\tilde{\xi}_{j}\right)$ are, for $j \geq 1$, independent sequences of independent random variables with $\xi_{j}$ and $\tilde{\xi}_{j}$ each being distributed according to the symmetric Cauchy distribution with parameter $\left(y_{j}-y_{j-1}\right)$. The sequence listed first in the lemma has $(2 j)$ th term given by $\sum_{i=0}^{j} \xi_{i}-\sum_{i=0}^{j} \tilde{\xi}_{j}$ and $(2 j-1)$ st term given by $\sum_{i=0}^{j} \xi_{i}-\sum_{i=0}^{j-1} \tilde{\xi}_{i}$. By the symmetry of the Cauchy distribution we may replace $\sum_{i=0}^{j} \tilde{\xi}_{j}$ with $-\sum_{i=0}^{j} \tilde{\xi}_{j}$ without altering the distribution of these variables. Similarly, the second sequence listed in the statement of the lemma has under $P^{x}$ the distribution of a sum of independent Cauchy random variables plus the initial value $x$. The proof is completed by comparing the parameter values of the two sequences, using the fact that if $X$ and $Y$ are independent symmetric Cauchy variables with parameter values $\alpha$ and $\beta$ respectively, then $X+Y$ is a symmetric Cauchy variable with parameter $\alpha+\beta$.

Using the lemma we have

$$
\begin{aligned}
\int_{\mathbf{R}^{n}} \tilde{E}^{0} E^{x}\left|\sum_{i=0}^{N-1}(\Delta u)_{i}\right|_{B}^{p} d x \\
\quad=\int_{\mathbf{R}^{n}} E^{x}\left|\sum_{i=0}^{N-1}\left[u\left(X\left(2 y_{i}\right), 2 y_{i}\right)-u\left(X\left(y_{i}+y_{i+1}\right), y_{i}+y_{i+1}\right)\right]\right|_{B}^{p} d x
\end{aligned}
$$


Now set $z_{2 i}=2 y_{i}, z_{2 i+1}=y_{i}+y_{i+1}, \varepsilon_{2 i}=1$ and $\varepsilon_{2 i+1}=0$ for $i=0, \ldots, N-1$.

Using this notation we have

$$
\begin{aligned}
& \int_{\mathbf{R}^{n}} \tilde{E}^{0} E^{x}\left|\sum_{i=0}^{N-1}(\Delta u)_{i}\right|_{B}^{p} d x \\
& =\int_{\mathbf{R}^{n}} E^{x}\left|\sum_{j=0}^{2 N-2} \varepsilon_{j}\left[u\left(X\left(z_{j}\right), z_{j}\right)-u\left(X\left(z_{j+1}\right), z_{j+1}\right)\right]\right|_{B}^{p} d x \\
& =\int_{\mathbf{R}^{n}} \int_{\mathbf{R}^{n}} h_{x}\left(x^{\prime}, A\right) E_{\left(x^{\prime}, A\right)}^{\prime x}\left|\sum_{j=0}^{2 N-2} \varepsilon_{j}\left[u\left(X\left(z_{j}\right), z_{j}\right)-u\left(X\left(z_{j+1}\right), z_{j+1}\right)\right]\right|_{B}^{p} d x d x^{\prime} \\
& =\int_{\mathbf{R}^{n}} E_{\left(x^{\prime}, A\right)}\left|\sum_{j=0}^{2 N-2} \varepsilon_{j}\left[u\left(X\left(z_{j}\right), z_{j}\right)-u\left(X\left(z_{j+1}\right), z_{j+1}\right)\right]\right|_{B}^{p} d x^{\prime} .
\end{aligned}
$$

By (2.1) the last expression is dominated by

$$
\begin{aligned}
& c_{p} \int_{\mathbf{R}^{n}} E_{\left(x^{\prime}, A\right)}\left|\sum_{j=0}^{2 N-2}\left[u\left(X\left(z_{j}\right), z_{j}\right)-u\left(X\left(z_{j+1}\right), z_{j+1}\right)\right]\right|_{B}^{p} d x^{\prime} \\
& \quad=c_{p} \int_{\mathbf{R}^{n}} E_{\left(x^{\prime}, A\right)}\left|f(X(0))-u\left(X\left(z_{2 N-1}\right), z_{2 N-1}\right)\right|_{B}^{p} d x^{\prime} \\
& \leq 2^{p} c_{p} \int_{\mathbf{R}^{n}} E_{\left(x^{\prime}, A\right)}|f(X(0))|_{B}^{p} d x^{\prime} .
\end{aligned}
$$

The last inequality follows from the fact that $|u(s, y)|_{B}^{p}$ is subharmonic and hence

$$
E_{\left(x^{\prime}, A\right)}\left|u\left(X\left(z_{2 N-1}\right), z_{2 N-1}\right)\right|_{B}^{p} \leq E_{\left(x^{\prime}, A\right)}|f(X(0))|_{B}^{p} .
$$

Finally, since $X(0)$ has $h_{x}\left(x^{\prime}, A\right) d x$ as its density function, we have

$$
\begin{aligned}
\int_{\mathbf{R}^{n}} E_{\left(x^{\prime}, A\right)}|f(X(0))|_{B}^{p} d x^{\prime} & =\int_{\mathbf{R}^{n}} \int_{\mathbf{R}^{n}} h_{x}\left(x^{\prime}, A\right)|f(x)|_{B}^{p} d x^{\prime} d x \\
& =\int_{\mathbf{R}^{n}}|f(x)|_{B}^{p} d x,
\end{aligned}
$$

and the proof is complete.

PROOF OF THEOREM 1.2. We shall sketch the proof since it is similar in many respects to the proof of Theorem 1.1; moreover, we limit ourselves to the one-dimensional case.

Let $m(\lambda)$ satisfy (1.3). We may assume without loss of generality that $m$ is smooth and supported in $[1,2]$. Let $\mu(\lambda)$ be the multiplier defined by

$$
\mu(\lambda)=|\lambda|^{2} \int_{1}^{2} e^{-|\lambda| y} y d y
$$

This multiplier is a member of the class of multipliers of Laplace transform type which has been extensively studied (see, e.g., [23 and 24]). If $a(y)$ is a bounded Riemann integrable function on $[0, \infty)$, let $\mu(a, k)(\lambda)=(-|\lambda|)^{k} \int_{0}^{\infty} e^{-|\lambda| y} y^{k-1} a(y) d y$. 
Let $f \in S_{F}(\mathbf{R} ; B)$ with Poisson integral $u(x, y)$ on $\mathbf{R}_{+}^{2}$. Then [23]

$$
T_{\mu(a, k)} f(x)=\int_{0}^{\infty} y^{k-1} u^{(k)}(x, y) a(y) d y .
$$

Specializing this formula to $k=2$ and $a(y)=1_{[1,2]}(y)$ we obtain

$$
T_{\mu} f(x)=\int_{1}^{2} y u_{y y}(x, y) d y
$$

for all $f \in S_{F}(\mathbf{R} ; B)$

Choose a smooth function $\sigma(\lambda)$ supported in $\left[\frac{1}{2}, 4\right]$ with $\sigma(\lambda)=1$ for $1 \leq \lambda \leq 2$. It is then easily seen that the multiplier $\sigma(\lambda) / \mu(\lambda)$ satisfies (3.1); hence by Theorem 1.1 we have that, for any $g \in L^{p}(\mathbf{R} ; B)$,

$$
\left\|T_{\sigma / \mu} g\right\|_{p} \leq c_{p}\|g\|_{p}, \quad 1<p<\infty .
$$

In particular, for $f \in D_{F}(\mathbf{R} ; B)$ the choice of $g=T_{\mu} T_{m} f$ yields

$$
\left\|T_{m} f\right\|_{p} \leq c_{p}\left\|T_{\mu} T_{m} f\right\|_{p} .
$$

Let $u, w$ and $v$ denote respectively the Poisson integrals of $f, T_{m} f$, and of the inverse Fourier transform of $m$. By (3.12), a change of variable, and a use of (3.4) as in the proof of Theorem 1.1 we obtain

$$
T_{\mu} T_{m} f(x)=16 \int_{1 / 4}^{1 / 2} \int_{-\infty}^{\infty} y v_{y}(s, y) u_{y}(x-s, 3 y) d s d y .
$$

Proceeding as above, introduce partitions of $\left[\frac{1}{4}, \frac{1}{2}\right]$ by $\frac{1}{4}=y_{0}<y_{1}<\cdots<y_{N}=$ $A=\frac{1}{2}$ and define $g_{N}(x)$ by

$$
g_{N}(x)=\sum_{i=0}^{N-1} \int_{-\infty}^{\infty} y_{i} v_{y}\left(s, y_{i}\right)\left[u\left(x-s, y_{i}+2 y_{i+1}\right)-u\left(x-s, 3 y_{i}\right)\right] d s .
$$

Put $e(s, y)=y v_{y}(s, y) h_{0}^{-1}(s, y)$. It is not true that $e(s, y)$ is uniformly bounded under the present hypotheses on $m$. Rather, we have the following result which replaces Lemma 3.1.

LEMMA 3.3. There is a constant $c$ depending only on $m$ such that

$$
\tilde{E}^{0}\left(\sup _{0 \leq i \leq n} e\left(\tilde{X}\left(y_{i}\right), y_{i}\right)\right)^{2} \leq C .
$$

(For the definition of $\tilde{E}^{0}$ and $\tilde{X}$ see the proof of Theorem 1.1.)

ProOF. Let

$$
\eta_{i}=\left|v_{y}\left(\tilde{X}\left(y_{N-i}\right), y_{N-i}\right)\right| h_{0}^{-1}\left(\tilde{X}\left(y_{N-i}\right), y_{N-i}\right) .
$$

For any $x^{\prime} \in \mathbf{R}$ and $A>0$ the sequence $\left(\eta_{i}\right)$ forms a submartingale under $\tilde{P}_{\left(x^{\prime}, A\right)}^{/ 0}$. This follows from (2.4) and the strong Markov property of Brownian motion. Therefore the sequence $\left(\eta_{i}\right)$ also forms a submartingale under $\tilde{P}^{0}$. Since $\frac{1}{4} \leq y_{i} \leq \frac{1}{2}$ we have, by Doob's maximal inequality,

$$
\tilde{E}^{0}\left(\sup _{0 \leq i \leq N} e\left(\tilde{X}\left(y_{i}\right), y_{i}\right)\right)^{2} \leq 8 \tilde{E}^{0} e\left(\tilde{X}\left(\frac{1}{4}\right), \frac{1}{4}\right)^{2} .
$$


The proof is completed by showing that the right side of the last inequality is bounded by a constant. In fact more is true: there is a constant $c$ such that

$$
\sup _{0<y<\infty} \tilde{E}^{0} e(\tilde{X}(y), y)^{2} \leq c,
$$

and this holds even if the assumption that $m$ is supported in $[1,2]$ is dropped. To see this use the fact that $\tilde{X}(y)$ has density function $h_{0}(s, y)$ and Plancherel's identity to obtain

$$
\begin{aligned}
\tilde{E}^{0} e(\tilde{X}(y), y)^{2} & =y \int_{-\infty}^{\infty}\left(s^{2}+y^{2}\right) v_{y}^{2}(s, y) d s \\
& =y \int_{-\infty}^{\infty}\left(\frac{d}{d \lambda}|\lambda| m(\lambda) e^{-|\lambda| y}\right)^{2}+\left(y|\lambda| m(\lambda) e^{-|\lambda| y}\right)^{2} d \lambda
\end{aligned}
$$

The assumption (1.3) on $m$ easily implies that the last expression is bounded by a constant independent of $y$, which completes the proof of Lemma 3.3.

We devote the rest of the proof to obtaining the inequality

$$
\left\|g_{N}\right\|_{p} \leq c_{p}\|f\|_{p}
$$

with $c_{p}$ independent of $N$, for $p$ in the range $[2, \infty)$. Passage to the limit as $N$ tends to infinity yields (1.11) for the same range of $p$, and a standard duality argument shows that (1.11) holds as well for $1<p \leq 2$. ( $B^{*}$ is $\zeta$-convex whenever $B$ is.)

As in the proof of Theorem 1.1 we have the following probabilistic representation of $g_{N}(x)$ :

$$
g_{N}(x)=E^{x} \sum_{i=0}^{N-1} \tilde{E}^{0} e\left(\tilde{X}\left(y_{i}\right), y_{i}\right)(\Delta u)_{i}
$$

where

$$
(\Delta u)_{i}=u\left(X\left(y_{i+1}\right)-\tilde{X}\left(y_{i}\right), y_{i}+y_{i+1}\right)-u\left(X\left(y_{i}\right)-\tilde{X}\left(y_{i}\right), 2 y_{i}\right)
$$

Let $e^{*}=\sup _{0 \leq i \leq N}\left|e\left(\tilde{X}\left(y_{i}\right), y_{i}\right)\right|$ and define a sequence $\varepsilon_{i}$ by $e^{*} \varepsilon_{i}=e\left(\tilde{X}\left(y_{i}\right), y_{i}\right)$ so that $\left|\varepsilon_{i}\right| \leq 1$. By Jensen's and Schwartz's inequalities,

$$
\begin{aligned}
\int_{-\infty}^{\infty}\left|g_{N}(x)\right|_{B}^{p} d x & \leq \int E^{x}\left|\sum_{i=0}^{N-1} \tilde{E}^{0} e^{*} \varepsilon_{i}(\Delta u)_{i}\right|_{B}^{p} d x \\
& \leq \int E^{x}\left(\tilde{E}^{0} e^{* 2}\right)^{p / 2} \tilde{E}^{0}\left|\sum_{i=0}^{N-1} \varepsilon_{i}(\Delta u)_{i}\right|_{B}^{p} d x \\
& \leq c_{(3.13)}^{p / 2} \int E^{x} \tilde{E}^{0}\left|\sum_{i=0}^{N-1} \varepsilon_{i}(\Delta u)_{i}\right|_{B}^{p} d x
\end{aligned}
$$

The proof is now completed exactly as in the proof of Theorem 1.1 since $\left|\varepsilon_{i}\right| \leq 1$ and $(\Delta u)_{i}$ is, for fixed values of the $\tilde{X}\left(y_{i}\right)$, a martingale difference sequence relative to $P_{\left(x^{\prime}, A\right)}$ for each $x^{\prime} \in \mathbf{R}$.

REMARK 3.1. The Hilbert transform in one dimension is obtained from a multiplier transform with $m(\lambda)=i \operatorname{sign}(\lambda)$. The corresponding function $y v_{y}(s, y) h_{0}^{-1}(s, y)$ then reduces to $2 s y /\left(s^{2}+y^{2}\right)$ which is bounded in absolute value 
by one. Thus a modification of the argument above leads to an independent proof of the main result of $[6]$.

REMARK 3.2. In the real case $(B=\mathbf{R})$ a modification of the proof of Theorem 1.2 gives a new proof of the multiplier theorem of Hörmander (Theorem $\mathrm{A}$ ).

If $\left(d_{k}\right)$ is a real-valued martingale difference sequence relative to some sequence of $\sigma$-fields then the square function, defined by $S_{n}=\left(\sum_{k=0}^{n} d_{k}^{2}\right)^{1 / 2}$, satisfies the two-sided inequalities

$$
c_{p}\left\|S_{n}\right\|_{p} \leq\left\|\sum_{k=0}^{n} d_{k}\right\|_{p} \leq C_{p}\left\|S_{n}\right\|_{p}
$$

for all $n$ and $1<p<\infty$ (see [4]).

Let $m$ be a multiplier defined on $\mathbf{R}$ and satisfying (1.3). We do not assume that $m$ is supported in some dyadic interval. Fix $p \geq 2$ and define $g_{N}$ as in the proof of Theorem 1.1 but with $y^{2} v_{y y}(s, y)$ replaced by $y v_{y}(s, y)$. Let $e(s, y)=$ $y v_{y}(s, y) h_{0}^{-1}(s, y)$. It is straightforward to check that both $\tilde{E}^{0} e\left(\tilde{X}\left(y_{i}\right), y_{i}\right)(\Delta u)_{i}$ and $\tilde{E}^{0}(\Delta u)_{i}$ are martingale difference sequences under $P_{\left(x^{\prime}, A\right)}$ for each $x^{\prime} \in \mathbf{R}$. Also note that the proof of Lemma 3.3 gives the estimate

$$
\tilde{E}^{0} e(X(y), y)^{2} \leq c, \quad y>0,
$$

with $c$ depending only on $m$. Using Jensen's inequality and an argument similar to that in the proof of Theorem 1.1 we have

$$
\left\|g_{N}\right\|_{p}^{p} \leq \int_{-\infty}^{\infty} E_{\left(x^{\prime}, A\right)}\left|\sum_{i=0}^{N-1} \tilde{E}^{0} e\left(\tilde{X}\left(y_{i}\right), y_{i}\right)(\Delta u)_{i}\right|^{p} d x^{\prime} .
$$

By Schwartz's inequality, (3.15) and (3.16),

$$
\left\|g_{N}\right\|_{p}^{p} \leq c \int_{-\infty}^{\infty} E_{\left(x^{\prime}, A\right)}\left(\sum_{i=0}^{N-1} \tilde{E}^{0}(\Delta u)_{i}^{2}\right)^{p / 2} d x^{\prime} .
$$

Since $p \geq 2$, Jensen's inequality and (3.15) yield

$$
\left\|g_{N}\right\|_{p}^{p} \leq c \int_{-\infty}^{\infty} E_{\left(x^{\prime}, A\right)} \tilde{E}^{0}\left|\sum_{i=0}^{N-1}(\Delta u)_{i}\right|^{p} d x^{\prime} .
$$

The proof is completed by using Lemma 3.2 exactly as in the proof of Theorem 1.1.

PROOF OF THEOREM 1.3. For convenience we limit ourselves to the onedimensional case. Also, by the boundedness of the Hilbert transform on $L^{p}(\mathbf{R} ; B)$ for $B \zeta$-convex we may assume without loss of generality that $\hat{f}(\lambda)=0$ for $\lambda<0$.

Choose a smooth function $\phi$ supported in $[1,2]$ with $\phi\left(\frac{3}{2}\right)=1$. Let $\psi$ supported in $\left[\frac{3}{4}, \frac{3}{2}\right]$ be defined by $\psi(\lambda)=1-\phi(\lambda)$ for $1 \leq \lambda \leq \frac{3}{2}$ and $\psi(\lambda)=1-\phi(2 \lambda)$ for $\frac{3}{4} \leq \lambda \leq 1$. Define multipliers $m_{1}(\lambda)$ and $m_{2}(\lambda)$ by

$$
m_{1}(\lambda)=\sum_{j=-\infty}^{\infty} \phi\left(2^{2 j}|\lambda|\right) \quad \text { and } \quad m_{2}(\lambda)=\sum_{j=-\infty}^{\infty} \phi\left(2^{2 j+1}|\lambda|\right) .
$$


Define similarly $m_{3}$ and $m_{4}$ by replacing $\phi$ with $\psi$. Each of the functions $m_{i}$ satisfies (3.1) and we have $m_{1}(\lambda)+\cdots+m_{4}(\lambda)=1$ for all $\lambda \neq 0$. It then follows that we have

$$
\|g\|_{p} \leq \sum_{i=1}^{4}\left\|T_{m_{i}} g\right\|_{p}
$$

for all $1<p<\infty$ and $g \in L^{p}(\mathbf{R} ; B)$. Let $m(\lambda)$ be a multiplier of the form

$$
m(\lambda)=\sum_{j=-\infty}^{\infty} \varepsilon_{j} 1_{\left[2^{j}, 2^{j+1}\right)}(\lambda)
$$

where $\varepsilon_{j} \in\{ \pm 1\}$. Note that $T_{(\varepsilon)}=T_{m}$. Since $m^{2}=1$ on $\mathbf{R}_{+}$it is sufficient to prove only the right-hand side of (1.13). We will show that there are constants $c_{p}$ such that

$$
\left\|T_{m_{1}} T_{m} f\right\|_{p} \leq c_{p}\|f\|_{p}
$$

for $1<p<\infty$ and for $f \in S(\mathbf{R} ; B)$. The same proof will show that (3.18) holds with $m_{1}$ replaced by each $m_{i}$ and the proof is then finished in view of (3.17) with $g=T_{m} f$.

Let $\left(\xi_{j}\right)_{-\infty<j<\infty}$ be a family of independent random variables defined on some probability space $(\Omega, \mathcal{F}, P)$ and each assuming the values \pm 1 with equal probability. Choose a smooth function $\rho$ supported in $\left[\frac{1}{2}, 4\right]$ and satisfying $\rho(\lambda)=1$ for $1 \leq \lambda \leq$ 2. For each $\omega \in \Omega$ define a multiplier $M(\omega, \lambda)=\sum_{j=-\infty}^{\infty} \xi_{j}(\omega) \rho\left(2^{2 j}|\lambda|\right)$. Clearly $M(\omega, \lambda)$ satisfies (3.1) with a constant independent of $\omega$. Also $|M(\omega, \lambda)|$ equals one on the support of $m_{1}$. Therefore we have, for any $1<p<\infty$ and $g \in L^{p}(\mathbf{R} ; B)$,

$$
\left\|T_{m_{1}} g\right\|_{p}^{p}=E\left\|T_{M} T_{m_{1}} g\right\|_{p}^{p}
$$

where $E$ denotes expectation with respect to the probability distribution of the $\xi_{j}$.

For each $\alpha \in \mathbf{R}$ define $H_{\alpha}(x)$ in terms of the Fourier transform by $\hat{H}_{\alpha}(\lambda)=$ $\operatorname{sign}(\lambda-\alpha)$. It follows at once from the boundedness of the Hilbert transform on $L^{p}(\mathbf{R} ; B)$ that we have

$$
\left\|H_{0} * g\right\|_{p} \leq c_{p}\|g\|_{p}
$$

for $g \in L^{p}(\mathbf{R} ; B)$. Also note that for each $H_{\alpha}$ we have $H_{\alpha} * g(x)=e^{i \alpha x} H_{0} *\left(e_{\alpha} g\right)(x)$, where $e_{\alpha}(x)=e^{-i \alpha x}$.

Let $\Phi_{j}$ denote the inverse Fourier transform of the function $\phi\left(2^{2 j}|\lambda|\right)$. Now for each $j$ there is some $\alpha_{j} \in \mathbf{R}$ and $\varepsilon_{j} \in\{ \pm 1\}$ such that

$$
\Phi_{j} * T_{m} f(x)=\varepsilon_{j} H_{\alpha_{j}} * \Phi_{j} * f(x)=\varepsilon_{j} e^{i \alpha_{j} x} H_{0} *\left(e_{\alpha_{j}} \Phi_{j} * f\right)(x) .
$$

Thus from (3.19) with $g=T_{m} f$ we obtain

$$
\left\|T_{m_{1}} T_{m} f\right\|_{p}^{p}=E \int_{-\infty}^{\infty}\left|\sum_{j=-\infty}^{\infty} \varepsilon_{j} \xi_{j} e^{i \alpha_{j} x} H_{0} *\left(e_{\alpha_{j}} \Phi_{j} * f\right)(x)\right|_{B}^{p} d x .
$$

The summands above form a martingale difference sequence on $(\Omega, \mathcal{F}, P)$ and $\left|\varepsilon_{j} e^{i \alpha_{j} x}\right| \leq 1$. Therefore, after interchanging the order of integration we obtain 
from (2.1)

$$
\begin{aligned}
\left\|T_{m_{1}} T_{m} f\right\|_{p}^{p} & \leq c_{p} E \int_{-\infty}^{\infty}\left|\sum_{-\infty}^{\infty} \xi_{j} H_{0} *\left(e_{\alpha_{j}} \Phi_{j} * f\right)(x)\right|_{B}^{p} d x \\
& =c_{p} E \int_{-\infty}^{\infty}\left|H_{0} *\left(\sum_{-\infty}^{\infty} \xi_{j} e_{\alpha_{j}} \Phi_{j} * f\right)(x)\right|_{B}^{p} d x .
\end{aligned}
$$

Using (3.20) and (2.1) again,

$$
\left\|T_{m_{1}} T_{m} f\right\| \leq c_{p} E \int_{-\infty}^{\infty}\left|\sum_{-\infty}^{\infty} \xi_{j} \Phi_{j} * f(x)\right|_{B}^{p} d x=c_{p} E\left\|T_{M} T_{m_{1}} f\right\|_{p}^{p} .
$$

Finally, we apply Theorem 1.1 twice to obtain (3.18).

The converse is an easy consequence of the result of Bourgain $[3]$.

\section{REFERENCES}

1. A. Benedek, A. P. Calderón and R. Panzone, Convolution operators on Banach space valued functions, Proc. Nat. Acad. Sci. U.S.A. 48 (1962), 356-365.

2. J. Bourgain, A generalization of a theorem of Benedek, Calderón, and Panzone, manuscript.

3. __ Remarks on Banach spaces in which martingale difference sequences are unconditional, Ark. Mat. 21 (1983), 163-168.

4. D. L. Burkholder, Martingale transforms, Ann. Math. Statist. 37 (1966), 1494-1504.

5. __ A geometrical characterization of Banach spaces in which martingale difference sequences are unconditional, Ann. Probab. 9 (1981), 997-1011.

6. D. L. Burkholder, A geometrical condition that implies the existence of certain singular integrals of Banach valued functions, Conf. on Harmonic Analysis in Honor of Antoni Zygmund (William Beckner, Alberto P. Calderón, Robert Fefferman and Peter W. Jones, eds.), Wadsworth, Belmont, Calif., 1983, pp. 270-286.

7. J. L. Doob, Conditional Brownian motion and the boundary limits of harmonic functions, Bull. Math. Soc. France 85 (1957), 431-458.

8. R. E. Edwards and G. I. Gaudry, Littlewood-Paley and multiplier theory, Springer-Verlag, New York, 1977.

9. R. F. Gundy and M. L. Silverstein, On a probabilistic interpretation for the Riesz transforms, Functional Analysis in Markov Processes, Lecture Notes in Math., vol. 923, Springer-Verlag, Berlin and New York, 1982.

10. R. F. Gundy and N. Th. Varopoulos, Les transformations de Riesz et les intégrales stochastiques, C. R. Acad. Sci. Paris 289 (1979), 13-16.

11. L. Hörmander, Estimates for translation invariant operators in $L^{p}$ spaces, Acta Math. 104 (1960) 93-139.

12. K. Itô and H. P. McKean, Jr., Diffusion processes and their sample paths, Springer-Verlag, Berlin and New York, 1974.

13. S. Kwapień, Isomorphic characterizations of inner product spaces by orthogonal series with vector valued coefficients, Studia Math. 44 (1972), 583-595.

14. J. E. Littlewood and R. E. A. C. Paley, Theorems on Fourier series and power series. I, J. London Math. Soc. 6 (1931), 230-233.

15. _ Theorems on Fourier series and power series. II, Proc. London Math. Soc. 42 (1936), 52-89.

16. __ Theorems on Fourier series and power series. III, Proc. London Math. Soc. 43 (1937), 105-126.

17. S. G. Mihlin, On the multipliers of Fourier integrals, Dokl. Akad. Nauk SSSR 109 (1956), 701703. (Russian)

18. J. Neveu, Discrete parameter martingales, North-Holland, Amsterdam, 1975.

19. G.-C. Rota, An "Altermeirende Verfahren" for general positive operators, Bull. Amer. Math. Soc. 68 (1962), 95-102. 
20. R. Salem and A. Zygmund, On lacunary trigonometric series, Proc. Nat. Acad. Sci. U.S.A. 33 (1947), 333-338.

21. M. J. Sharpe, Some transformations of diffusions by time reversal, Ann. Probab. 8 (1980), 11571162.

22. E. M. Stein, Singular integrals and differentiabritity properties of functions, Princeton Univ. Press, Princeton, N.J., 1970.

23. __ Topics in harmonic analysis related to the Littlewood-Paley theory, Princeton Univ. Press, Princeton, N.J., 1970.

24. N. Varopolous, Aspects of probabrilistic Littlewood-Paley theory, J. Funct. Anal. 38 (1980), 25-60.

Department of Mathematics, Cornell University, IthaCA, NeW York 14853

Current address: Department of Mathematics, Syracuse University, Syracuse, New York 13210 